\title{
Effect of Plasma Actuator on Flow Field
}

\author{
L. Yue, Y.F. WanG*, Y.W. Zhang And X. ZhaO \\ School of Transportation and Vehicle Engineering, Shandong University of Technology, \\ No. 266 Xincun West Road, 255049, Zibo, China
}

Received: 05.11.2021 \& Accepted: 23.11.2021

Doi: $10.12693 /$ APhysPolA.141.74

*e-mail: wangyunfei@sdut.edu.cn

\begin{abstract}
In order to study the influence of the plasma actuator on the air flow in more detail, the effect of the plasma actuator on the flow field was simplified to the volume force of the electric field. Reynolds Average Navier-Stokes method and transition SST turbulence model were used to simulate the air flow field at different loading voltage, power frequency and inflow velocity. The results show that the higher the loading voltage and power frequency of the plasma actuator, the stronger the effect of the actuator on the flow field. The induced velocity increases non-linearly with increasing loading voltage, however, the increasing rate is getting slower and slower. When the voltage rises to about $30 \mathrm{kV}$, the acceleration effect of the actuator to air reaches the limit. The induced velocity increases linearly with the increase of the power frequency, but the change of the power frequency does not affect the operating range of the actuator in the vertical direction. Provided that parameters such as loading voltage and power frequency are constant, the higher the inflow velocity, the weaker the actuator's effect on the flow field. And the higher velocity inflow obviously suppresses the range of the actuator in the vertical direction.
\end{abstract}

topics: plasma actuator, phenomenological model, numerical simulation, induced velocity

\section{Introduction}

With the continuous development of active fluid control technology, dielectric barrier discharge (DBD) plasma actuators have gradually attracted wide attention from researchers worldwide [1-9]. With the advantages of a lightweight, small volume, low cost and fast reaction, it has been gradually applied to the fields of boundary layer separation control, transition flow control and turbulent flow control. Good control results have been obtained [10-13]. Researchers around the world attach great importance to the research of plasma active fluid control technology. The US Air Force regards plasma active fluid control technology as one of six basic research projects to keep future science and technology in the leading position in the world. Detailed research is carried out on the plasma improving aero-engine aerodynamic characteristics and work efficiency [14]. In 2004, the US Department of Defense listed plasma flow-control technology as an important direction of national aerospace development, and laid the scientific and theoretical foundation of the new generation of aerospace revolution by accurately understanding the effect of plasma [15]. In 2005, China listed plasma dynamics as "Major Mechanical Problems In Aerospace". In 2009, the American Academy of Aeronautics and Astronautics listed plasma flow control technology as one of the top ten aerospace cutting-edge technologies.
In October 2019, an important research project on plasma active fluid control called "Plasmaero (Useful Plasma For Aerodynamic Control)" was carried out in Europe [16]. Therefore, strengthening the research on the plasma actuator of dielectric barrier discharge (DBD) is of great significance for improving the strength of aerospace industry and strengthen the theoretical basis of scientific research.

A schematic diagram and the phenomenological model of the plasma actuator are shown in Figs. 1 and 2, respectively. Two electrodes are asymmetrically arranged, one is exposed to air, the other is encapsulated in an insulating medium. The two electrodes are separated by a resin plate. When high-frequency and high-voltage alternating current is applied to the two electrode plates, a strong electric field will accelerate electrons and ions in the air near the electrode and collide with neutral molecules and atoms such as oxygen and nitrogen. When the electron energy becomes large enough, the electron will dissociate, ionize neutral particles and then release a new electron. New electrons continue to collide with neutral particles, ionizing them until an avalanche of electrons breaks through the air. Electrons and ions make a directional motion in the electric field, collide with neutral particles, and transfer their momentum and energy to the surrounding air. Hence, the air near the exposed electrode produces directional motion, forming an air jet. 


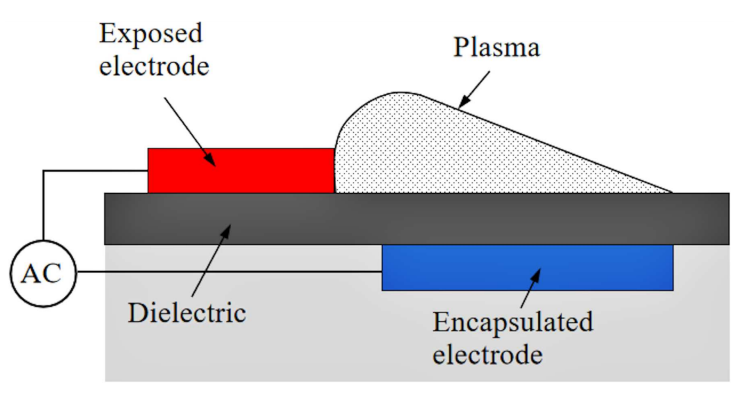

Fig. 1. Schematic diagram of plasma actuator.

Roy et al. [17] used Reynolds Average NavierStokes (RANS) method to study the induced flow field of the plasma actuator. Audier et al. [18-21] indicated that the plasma discharge significantly affects the turbulent kinetic energy, and a positive discharge induces a faster ionic wind than a negative discharge. The effect of strip actuators on film cooling was also investigated there. The results show that the effectiveness can be increased by the plasma discharge whatever the blowing ratio. Yang Guoqing et al. [22-24] studied the characteristics of dielectric and carried out related experiments on a gas-liquid two-phase plasma actuator compared to a traditional plasma actuator (Fig. 2). The results show that the discharge power of the former is obviously greater than that of the latter under the same experimental conditions. The discharge power of the gas-liquid two-phase plasma actuator is quite different at different air humidity, and the maximum discharge power appears at a specific air humidity. Yi Chen et al. [25] designed a plasmaassisted combustion actuator to improve the performances of an aero-engine combustion chamber. Experimental results show that discharge produces multiple species including $\mathrm{O}, \mathrm{O}_{3}, \mathrm{O}_{2}$ etc. Combustion efficiency is improved by plasma, and the higher the voltage, the more noticeable the increase of the combustion efficiency. Wilde et al. [26] developed a physics-based model for surface dielectric barrier discharges (SDBDs) power consumption which accounts for material and geometric variation between SDBDs. They measured the power of SDBDs of different materials, thicknesses and electrical operating points, and find a correlation between model and experiment. Sato et al. [27] developed a novel method for fast simulation of discharge, which uses a simple scalar equation to calculate the electric field variation. The results show that this method significantly reduces computation time and error. Xin Zhang et al. [28-30] studied the whole development process of the induced flow field from the starting point of the thin jet to the quasi-steady stage of wall jet.

The induction effect of plasma excitation has been studied by various methods, it has been widely used in areas such as active flow control and combustion control. However, understanding of the mechanism of the actuator is not perfect at present,

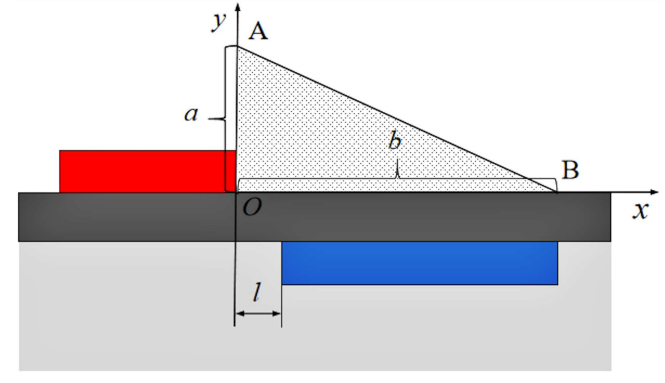

Fig. 2. Phenomenological model of plasma actuator.

selection of loading voltage and other parameters requires some experience. Its scope of application is limited. In addition, for the actuator with the parameter selected in this paper, the maximum voltage loaded on the plate is very limited and the induced air velocity is also low in the above study. There is no detailed summary and analysis of plasma induction characteristics when the loading voltage varies over a wide range. It is pointed out in some studies that the speed of air induced by the actuator is proportional to the change of voltage, but does it still satisfy this rule if the voltage continues to rise? If research studies on plasma control of flow in low-pressure turbines are carried out in the future, their aim will be to restrain the separation of the suction surface boundary layer and reduce the total pressure loss at cascade outlet. However, the Mach number at the outlet of the low-pressure turbine is high, the airflow velocity is about $10 \mathrm{~m} / \mathrm{s}$. It is necessary to increase the loading voltage and the power frequency of the actuator. In addition, it is important to understand the influence of the change of inflow velocity on the excitation effect.

In this paper, the effect of the plasma actuator on the flow field is simplified to electric field volume force, and the induced flow field is simulated by using the RANS method and transition SST turbulence model. The effect of the plasma actuator on the flow field under different loading voltage, power frequency and inflow velocity is studied. The maximum load voltage is up to $30 \mathrm{kV}$. It enriches the theory of plasma actuator mechanism and provides a reference for plasma control of high-speed gas flow.

\section{Phenomenological model and numerical simulation method}

In this paper, the effect of the plasma actuator on the flow field was simplified to the volume force of the electric field by using the theory of Shyy et al. [31]. It is assumed that the effect of the actuator on the flow field only exists in the triangular OAB region, and the air outside the region cannot be ionized. The maximum field strength $E_{\mathrm{O}}$ between the plates is the field strength at $\mathrm{O}$ point. It is calculated from formula $E_{\mathrm{O}}=V_{\mathrm{O}} / l$, where $V_{\mathrm{O}}$ is the 


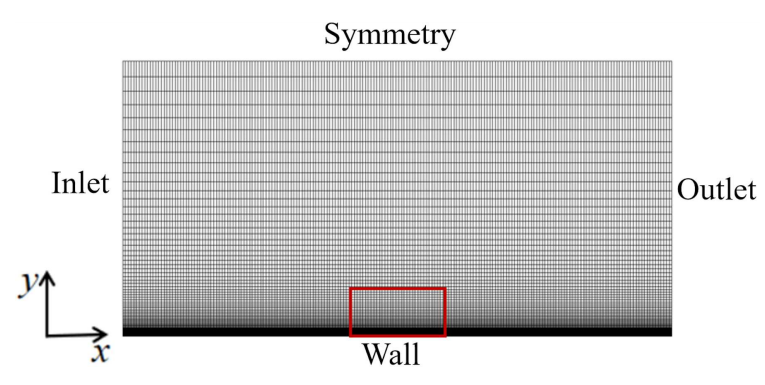

Fig. 3. Model structure and grid diagram.

loading voltage $(4-30 \mathrm{kV})$ root mean square (rms), and $l$ is the distance between the two electrodes $(l=0.25 \mathrm{~mm})$. The electric field strength in the triangular $\mathrm{OAB}$ decreases linearly with the distance from $\mathrm{O}$ point, and its internal electric field strength satisfies the formula $E=E_{\mathrm{O}}-k_{1} x-k_{2} y$. The breakdown threshold of the electric field strength is $E_{b}=30 \mathrm{kV} / \mathrm{cm}$. The electric field strength in $x$ and $y$ direction is as follows

$$
E_{x}=\frac{E k_{2}}{\sqrt{k_{1}^{2}+k_{2}^{2}}}
$$

and

$$
E_{y}=\frac{E k_{1}}{\sqrt{k_{1}^{2}+k_{2}^{2}}} .
$$

Here, $\quad k_{1}=\frac{1}{b}\left(E_{\mathrm{O}}-E_{b}\right), \quad k_{2}=\frac{1}{a}\left(E_{\mathrm{O}}-E_{b}\right)$, $a=0.0015 \mathrm{~m}, b=0.003 \mathrm{~m}, f$ is the power frequency, the electron density $\rho_{e}=1 \times 10^{17} / \mathrm{m}^{3}$, and $e$ is elementary charge. The plasma discharge time in an excitation cycle of $\mathrm{AC}$ power is $\Delta t=67 \mu \mathrm{s}$. The effect during other times of the cycle can be neglected as it has little or no plasma formation [31]. Therefore, the volume force components of the electric field in $x$ and $y$ direction are, respectively,

$$
\begin{aligned}
F_{x} & =e \rho_{e} E_{x} f \Delta t \\
\text { and } & \\
F_{y} & =e \rho_{e} E_{y} f \Delta t .
\end{aligned}
$$

The electric field volume force is coupled with the flow field by using the UDF function of Fluent. The DEFINE SOURCE macro command discretely solves the electric field volume force, and the electric field volume force is coupled to the momentum equation in the form of momentum source term.

A two-dimensional computational domain model is adopted in this paper. The transition SST turbulence model is sensitive to the factors such as turbulence, separation and pressure gradient. As shown in Fig. 3, the left and right sides of the computational domain are air inlet and airflow outlet, respectively. The boundary condition of the inlet is a velocity inlet and the outlet is a free flow outlet. The upper part of the computational domain is set as a symmetrical surface and the bottom is a nonslip wall. There are 100 nodes in the $x$ direction and 200 nodes in the $y$ direction, the grid near the wall is locally refined. The size of the first layer of the grid near the wall is $0.0001 \mathrm{~m}$, the number of global grids is about 20000. The medium is ideal compressible gas, and the inlet turbulence is $0.5 \%$.

\section{Results and discussion}

\subsection{Numerical method verification}

Figure 4 shows the contour of air velocity of the plasma-induced flow field and a comparison of calculated results and numerical simulation results by Shyy et al. [31]. The parameter values for the case are the same as [31]. The loading voltage is $4 \mathrm{kV}$, the power frequency is $3000 \mathrm{~Hz}$. Figure $4 \mathrm{a}$ shows that as the plasma actuator applies more momentum to the air, the air velocity downstream of the exposed electrode increases significantly and attenuates along the flow direction. Figure $4 \mathrm{~b}$ shows that the operating range of the plasma actuator in the vertical direction is about $2 \mathrm{~mm}$, and the peak velocity is about $6.2 \mathrm{~m} / \mathrm{s}$, which has a good effect on air in the boundary layer near the wall. The calculated results in this paper are consistent with the numerical simulation results by Shyy [31].

\subsection{Results analysis}

The velocity distribution of the plasma-induced flow field under different loading voltages in still air is shown in Fig. 5. The values of the power frequency

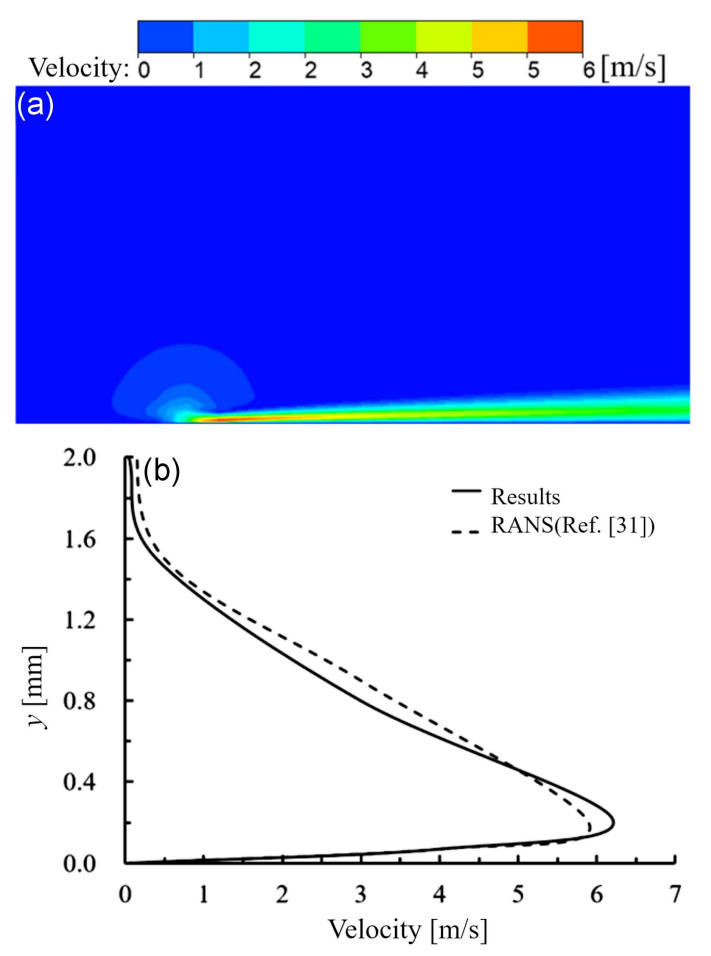

Fig. 4. (a) Velocity in the figure is the air flow rate. Diagram showing the contour of air velocity of plasma-induced flow field. (b) Calculated results and numerical simulation results [31] comparison. 


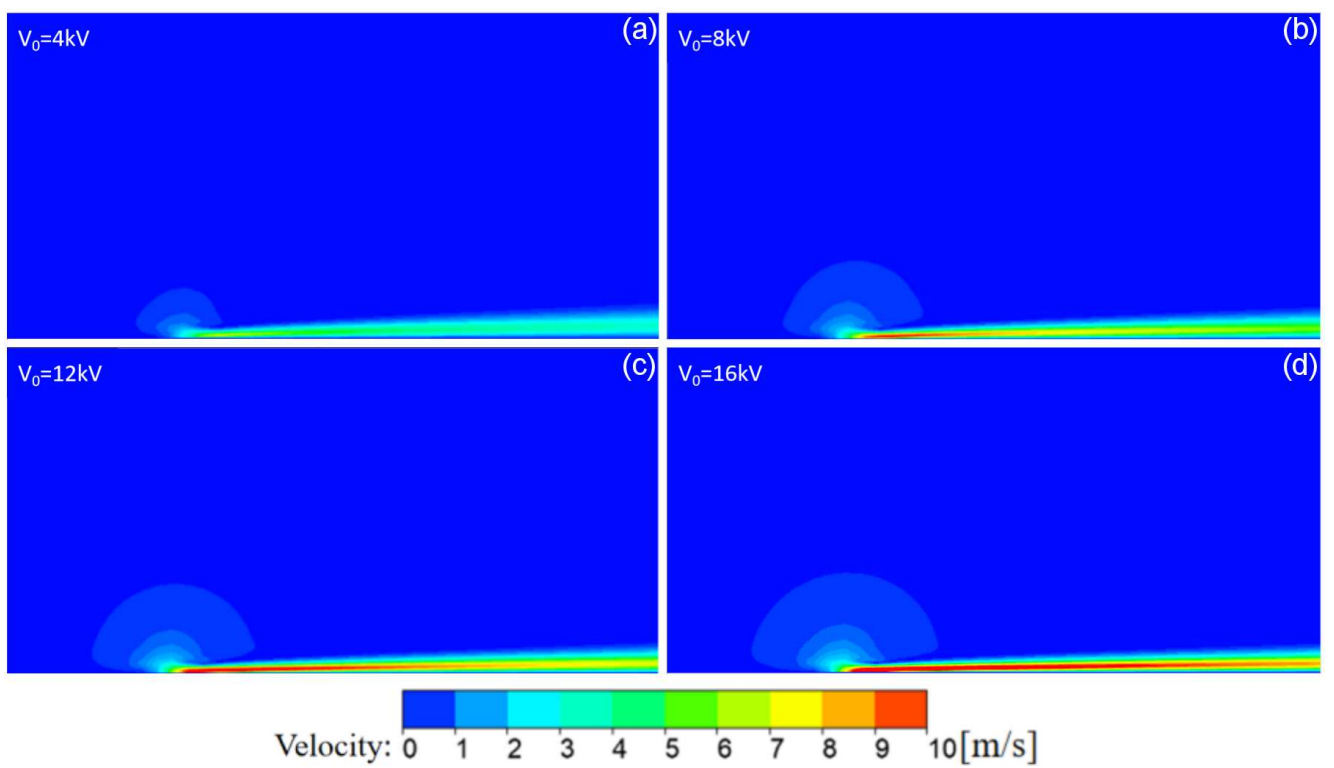

Fig. 5. Velocity distribution of flow field under different loading voltages.

for the different cases are the same. The power frequency is $3000 \mathrm{~Hz}$. The area shown is the position of the red mark in Fig. 3. With small differences in the velocity contour at the loading voltage above $16 \mathrm{kV}$, the phenomenon is not obvious. Due to the space limitation, the velocity contour at the loading voltage below $16 \mathrm{kV}$ is given. The disturbance range of the actuator in the flow field has little difference under different loading voltages, but the air velocity obviously increases with the increase of the loading voltage in the disturbance range. The air flow velocity at a distance of $2 \mathrm{~mm}$ from the bare electrode is shown in Fig. 6. Data in Fig. 6b are the peak air velocities under different loading voltages in Fig. 6a. The flow velocity of air decreases gradually in the $y$ direction, eventually falling to 0 . The disturbance range of each condition in the $y$ direction is basically the same, which is consistent with the phenomenon observed in Fig. 5. As the loading voltage increases gradually from $4 \mathrm{kV}$ to $30 \mathrm{kV}$, the peak air velocity increases gradually from $6.2 \mathrm{~m} / \mathrm{s}$ to $16.2 \mathrm{~m} / \mathrm{s}$. The slope of the curve gradually decreases, indicating a slower and slower rise. The change of air velocity is small for a voltage greater than $20 \mathrm{kV}$. With the voltage increasing by $10 \mathrm{kV}$, the peak air velocity only increases by $0.2 \mathrm{~m} / \mathrm{s}$. From this, we can infer that for the plasma actuator of this structure, the acceleration effect on the air has reached its limit when the voltage reaches $30 \mathrm{kV}$. If the voltage continues to rise, the control effect of a model continues to rise as well. We will consider whether it is due to the factors such as temperature and magnetic field.

For a more detailed study of the effect of the plasma actuator on the flow field under different inflow velocities, the velocity contour of the plasmainduced flow field is shown in Fig. 7. The loading voltage is $4 \mathrm{kV}$, the power frequency is $3000 \mathrm{~Hz}$,
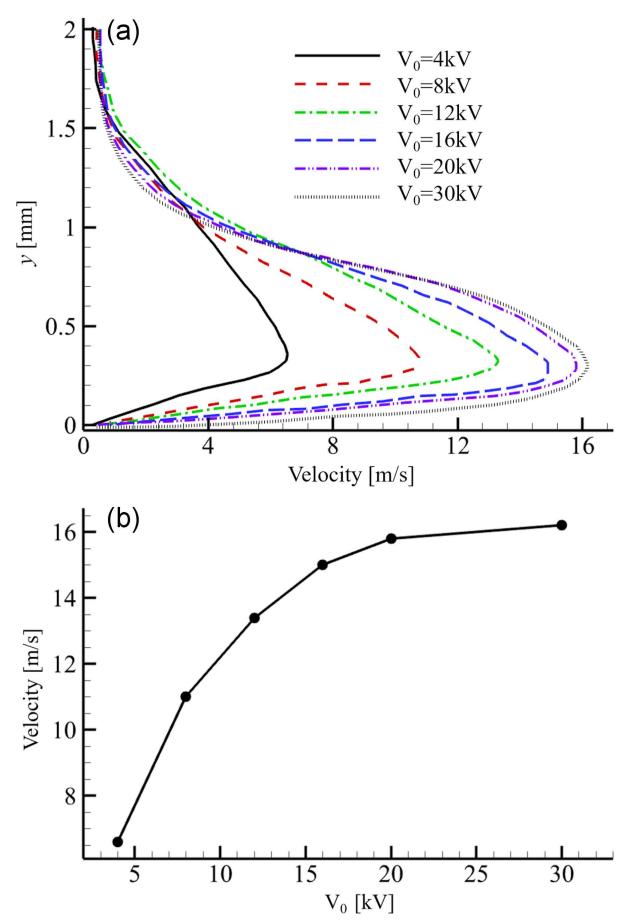

Fig. 6. (a) Diagram showing air velocity at the $2 \mathrm{~mm}$ position from the bare electrode under different loading voltages. (b) Peak air velocity under different loading voltages.

and the inflow velocity is $U=2 \mathrm{~m} / \mathrm{s}, 4 \mathrm{~m} / \mathrm{s}, 6 \mathrm{~m} / \mathrm{s}$ and $8 \mathrm{~m} / \mathrm{s}$. As can be seen in Fig. 7, the effect of the plasma actuator on the flow field is more evident at a lower inflow velocity. The velocity of the air near the actuator is obviously higher than the mainstream velocity. The high-speed gas induced by the plasma actuator gradually slows down in the flow direction, it tends to be consistent with the 


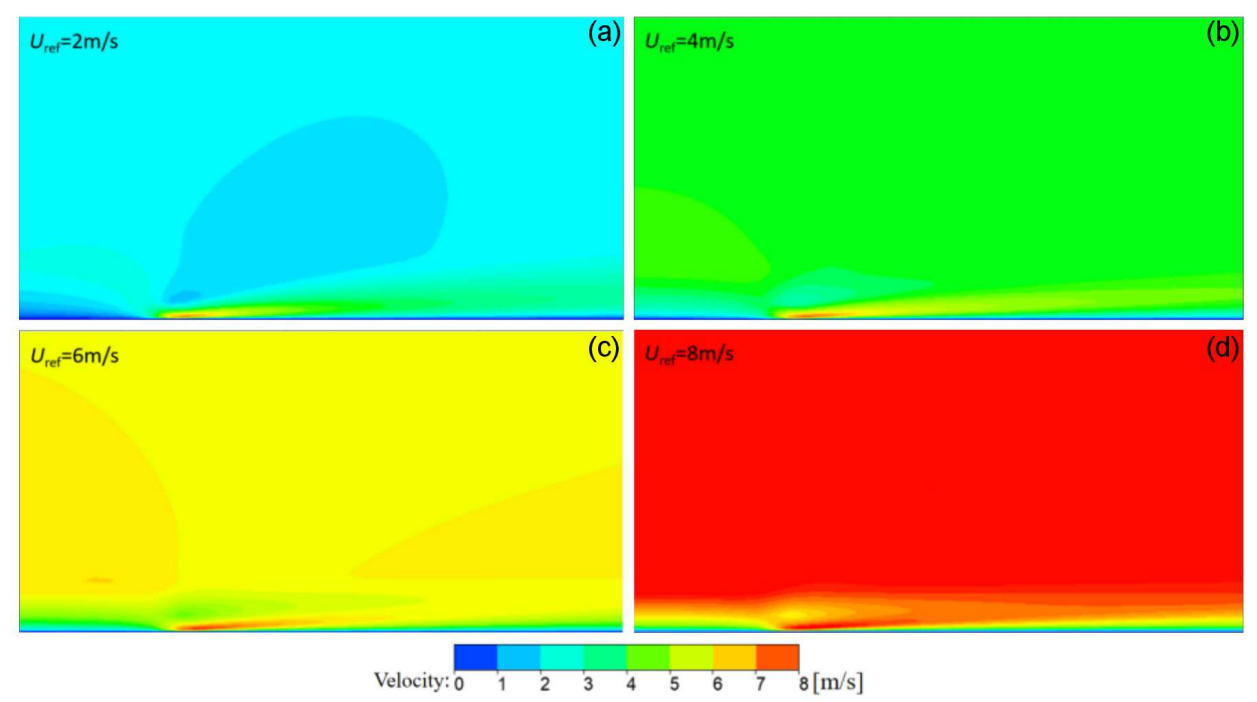

Fig. 7. Velocity distribution of flow field under different inflow velocities.
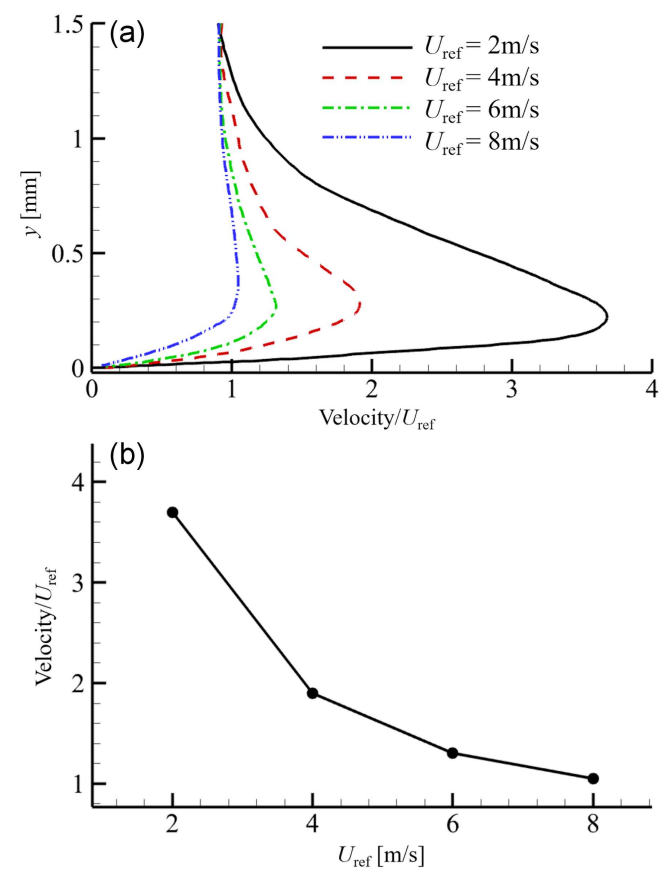

Fig. 8. (a) Comparison of air velocity at the $2 \mathrm{~mm}$ position from the bare electrode under different inflow velocities. (b) Peak air velocity under different inflow velocities.

mainstream. The disturbance range in the $y$ direction continues to increase along the flow direction. With the increase of the inflow velocity, the effect of the plasma actuator gradually decreases. When the mainstream velocity rises to $8 \mathrm{~m} / \mathrm{s}$, the air velocity induced by the actuator is essentially the same as the mainstream velocity. And the disturbance range in the $y$ direction also decreases with the increase of the inflow velocity.

Figure 8 shows the air flow velocity at the $2 \mathrm{~mm}$ position at the distance from the exposed electrode under different inflow velocities. The effect of the plasma actuator on the flow field is characterized by Velocity/ $U_{\text {ref. }}$ It can be seen in Fig. 8 that there is a peak point of velocity near the $y=0.3 \mathrm{~mm}$, indicating that the position of the peak point is independent of the inflow velocity. The effect of the plasma actuator on the flow field under low inflow velocity is obviously higher than that in other conditions. With the increase of the inflow velocity, the effect of the plasma actuator on the flow field decreases nonlinearly. The slope of the curve gradually decreases, indicating a slower and slower decreasing. It can be clearly seen in the figure that the induced air velocity is consistent with the mainstream velocity earlier under higher inflow velocity, indicating that higher-velocity inflow suppresses the disturbance of the actuator in the $y$ direction. It should be noted that, there is an obvious velocity deficit in the flow field under different inflow velocities. The magnitude of the deficit increases gradually with increasing of the inflow velocity. Then the air velocity along the $y$ direction tends to be consistent with the inflow velocity.

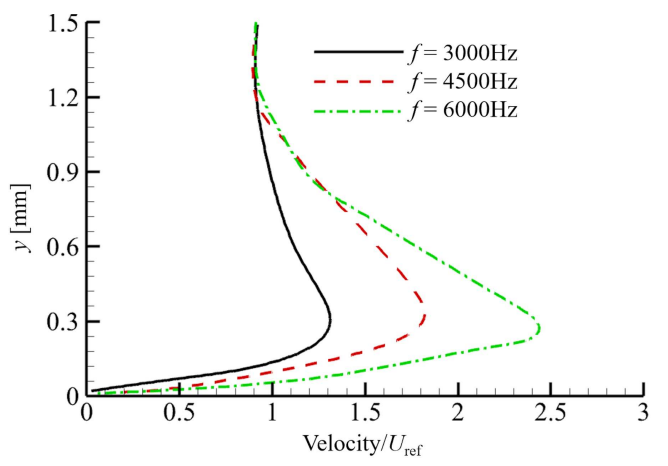

Fig. 9. Comparison of air velocity at the $2 \mathrm{~mm}$ position from the bare electrode under different power frequencies. 
Figure 9 shows the air velocity at the $2 \mathrm{~mm}$ position from the exposed electrode under different power frequencies. The loading voltage is $4 \mathrm{kV}$, and the inflow velocity is $6 \mathrm{~m} / \mathrm{s}$. It can be seen in Fig. 9 that the air velocity induced by the plasma actuator is higher at higher power frequency. The velocity increases linearly with the increase of frequency. For each increase in frequency by $1500 \mathrm{~Hz}$, the air velocity increases by $\approx 3 \mathrm{~m} / \mathrm{s}$. The peak air velocities are all located near $y=0.3 \mathrm{~mm}$. The air velocities decrease along the $y$ direction gradually, which are all consistent with the mainstream velocities near $y=1.2 \mathrm{~mm}$. Therefore, the actuation disturbance range in the $y$ direction is independent of the power frequencies.

\section{Conclusion}

- The higher the loading voltage and power frequency of the plasma actuator, the stronger the effect of the actuator on the flow field.

- The induced velocity increases nonlinearly with the increase of loading voltage, the increasing rate is getting slower and slower. When the voltage rises to about $30 \mathrm{kV}$, the acceleration effect of the actuator to the air reaches the limit.

- The induced velocity increases linearly with the increase of the power frequency, but the change of the power frequency does not affect the operating regime of the actuator in the vertical direction.

- Provided that the parameters such as loading voltage and power frequency are constant, the higher the inflow velocity, the weaker the effect of the actuator on the flow field. And the higher-velocity inflow obviously suppresses the disturbance of the actuator in the vertical direction.

\section{Acknowledgments}

This work was supported by the project ZR2020QE190 supported by Shandong Provincial Natural Science Foundation and The National Natural Science Foundation of China (No. 51906243).

\section{References}

[1] T.C. Corke, M.L. Post, D.M. Orlov, Prog. Aerosp. Sci. 43, 193 (2007).

[2] D.E. Ashpis, D.R. Thurman, Int. J. Turbo. 36, 157 (2019).

[3] E. Pescini, M.G. De Giorgi, A. Suma, L. Francioso, A. Ficarella, Aerosp. Sci. Technol. 76, 442 (2018).
[4] A. Hatamoto, K. Emori, H. Nishida, ASME Fluids Eng. Div. Publ. FEDSM. 3, 3 (2021).

[5] F. Kozo, Appl. Sci. 8, 546 (2018).

[6] A. Nakano, H. Nishida, J. Appl. Phys. 126, 173303 (2019).

[7] D.E. Ashpis, M.C. Laun, E.L. Griebeler, AIAA J. 55, 2254 (2017).

[8] H. Aono, H. Fukumoto, Y. Abe, M. Sato, T. Nonomura, K. Fujii, Energies 13, 1218 (2020).

[9] S. Karimi, B. Mohammadikalakoo, P. Schito, J. Wind Eng. Ind. Aerodyn. 209, 104488 (2021).

[10] T.C. Corke, C.L. Enloe, S.P. Wilkinson, Annu. Rev. Fluid Mech. 42, 505(2010).

[11] Xuanshi Meng, Ke Song, Yuexiao Long, Huaxing Li, Acta Aerodyn. Sin. 36, 901 (2018).

[12] W.S. Nie, Y.F. Cheng, X.K. Che, Adv. Mech. 42, 722 (2012).

[13] Z.S. Ni, G.Q. Li, C. Gao, Vacuum \& Cryogenics 20, 9 (2014).

[14] Y.H. Li, Y. Wu, J. Air Force Eng. Univ. (Nat. Sci. Ed.) 13, 1 (2012).

[15] Y. Wu, Y.H. Li, Acta Aeronaut. Astronaut. Sin. 36, 381 (2015).

[16] D. Caruana, Plasma Phys. Controlled Fusion 52, 124045 (2013).

[17] C.C. Wang, S. Roy, J. Appl. Phys. 104, 073305 (2008).

[18] P. Audier, M. Fénot, N. Bénard, E. Moreau, Appl. Phys. Lett. 108, 084103 (2016).

[19] P. Audier, M. Fénot, N. Bénard, E. Moreau, Int. J. Heat Fluid Flow. FL. 62, 247 (2016).

[20] E. Moreau, P. Audier, T. Orriere, J. Appl. Phys. 125, 133303 (2019).

[21] M. Fénot, P. Audier, B. Romain, Int. Symp. Turbul. Shear Flow Phenom. 2, 2 (2017).

[22] G.Q. Yang, S. Zhong, Q. Zhang, D.Y. Wang, Y.S. Cao, Gaodianya Jishu 43, 4000 (2017).

[23] G.Q. Yang, Y. Li, D.Y. Wang, J. Liu, J.X. Li, Diangong Jishu Xuebao 34, 1106 (2019).

[24] G.Q. Yang, Y. Guo, D.Y. Wang, S.C. Wang, R. Jia, P. Li, Gaodianya Jishu 43, 2825 (2017).

[25] Yi Chen, Li-Ming He, Li Fei, Jun Deng, Jian-Ping Lei, Han Yu, Aerosp. Sci. Technol. 99, 105765 (2020). 
[26] N.D. Wilde, H.F. Xu, N. Gomez-Vega, S.R.H. Barretta, Appl. Phys. Lett. 118, 154102 (2021).

[27] S. Sato, T. Shiroto, M. Takahashi, N. Ohnishi, Plasma Sources Sci. Technol. 29, 075007 (2020).

[28] X. Zhang, Y.D. Cui, C.M.J. Tay, B.C.Khoo, Aerosp. Sci. Technol. 99, 105751 (2020).
[29] X. Zhang, Y.D. Cui, C.M.J. Tay, B.C.Khoo, Chin. J. Aeronaut. 34, 13 (2021).

[30] P.Y. Yang, X. Zhang, C. Pan, Aerosp. Sci. Technol. 118, 107071 (2021).

[31] W. Shyy, B. Jayaraman, A. Andersson, J. Appl. Phys. 92, 6434 (2002). 